\title{
INTERACCIÓN DE AGLUTINANTES DE FLUORURO DE POLIVINILIDENO (PVDF) CON SOLUCIONES FUERTEMENTE ALCALINAS
}

\section{INTERACTION OF POLYVINYLIDENE FLUORIDE (PVDF)-BASED BINDERS WITH STRONGLY AlKALine SOLUTIONS}

\author{
Leonard Pagliaro ${ }^{1}$ y Daniel A. Lowy ${ }^{* 2}$ [i]
}

\author{
${ }^{1}$ Investigador Científico, FlexEl, LLC, College Park, MD 20740, USA \\ ${ }^{2}$ Northern Virginia Community College, Departamento de Ciencias e Ingeniería, Alexandria Campus, Alexandria, VA 22311, \\ USA
}

*Autor para correspondencia: daniellowy@gmail.com

\begin{abstract}
Resumen
Se propone una metodología para evaluar los fenómenos degradantes de aglutinantes en los medios alcalinos, información que debe de ser de gran ayuda para los expertos en el campo de la investigación y desarrollo de baterías, ya que se podría evaluar la estabilidad esperada de los aglutinantes fluorados antes de ser probados en células galvánica ensambladas. Por esto, se ahorra tiempo, esfuerzo y se agiliza el trabajo. Mientras que el poli (tetrafluoroetileno), PTFE, ha demostrado ser químicamente inerte, este plantea un desafío tecnológico severo, siendo difícil de procesar debido a su fibrilación. Los aglutinantes alternos son poli (1, 1-difluoroetileno), PVDF, y sus copolímeros, conocidos bajo el nombre comercial de Kynar®, que son significativamente más fáciles de usar. Sin embargo, cuando se mantiene en contacto con soluciones fuertemente alcalinas, la estabilidad química de Kynars se ve comprometida. Estos pueden someterse a reacciones de eliminación con la liberación de iones de fluoruro y la formación de enlaces dobles. Estos enlaces $\pi$ pueden degradarse aún más por oxidación, en contacto con oxidantes incorporados en el cátodo. Estos procesos químicos no deseados pueden inhibir las propiedades aglutinantes y, en última instancia, pueden reducir la vida útil de las células galvánica, agotando el rendimiento de la batería. En este rol, se investigó la descomposición de la aglutinación de PVDF en medios alcalinos siguiendo dos pasos: (i) los aglutinantes puros fueron probados exponiéndolos a $32 \mathrm{wt} \%$ solución acuosa de $\mathrm{KOH}$, a $60^{\circ} \mathrm{C}$, temperatura que acelera los procesos degradantes, y (ii) se investigaron los aglutinantes incorporados en la pasta cátodo en condiciones similares. La degradación del aglutinante se evaluó determinando la concentración de iones de fluoruro liberados, monitoreando el cambio de color y la formación de precipitados y grabando e interpretando los espectros FT-IR.
\end{abstract}

Palabras clave: Kynar, polivinílico (1,1- difluoretileno), PVDF, PTFE, deshidrohalogenación, aglutinante del cátodo, 
células galvánicas.

\begin{abstract}
A careful methodology to monitor binder degrading phenomena in alkaline media is proposed, which should assist experts in field of battery research and development, as it can evaluate the expected stability of fluorinated binders, prior to being tested in assembled galvanic cells. By this, it saves time, effort, and expedites work. While poly(tetrafluoroethylene), PTFE, has proven chemically inert, it poses a severe technological challenge, being difficult to process due to its fibrillation. Alternate binders are poly(1,1-difluoroethylene), PVDF, and its copolymers, known under the trade name of Kynar $\AA$, which are significantly easier to use. Nevertheless, when kept in contact with strongly alkaline solutions, the chemical stability of Kynars is compromised. They may undergo elimination reactions with the release of fluoride ions and the formation of double bonds. These $\pi$ bonds may further degrade by oxidation, in contact with oxidizers incorporated in the cathode. Such undesired chemical processes may inhibit the binding properties and, ultimately, may reduce shelf life of galvanic cells, depleting battery performance. In this paper PVDF binder decomposition in alkaline media was investigated in two steps: (i) pure binders were tested by exposing them to 32 wt \% aqueous $\mathrm{KOH}$ solution, at $60^{\circ} \mathrm{C}$, a temperature that accelerates degrading processes, and next (ii) binders incorporated in cathode paste were investigated under similar conditions. Binder degrading was evaluated by determining the concentration of released fluoride ions, monitoring color change and precipitate formation and recording and interpreting FT-IR spectra.

Keywords: Kynar, poly(1,1-difluoroethylene), PVDF, PTFE, dehydrohalogenation, cathode binder, galvanic cells.
\end{abstract}

Forma sugerida de citar: Pagliaro, L. y Lowy, D. A. (2019). Interacción de aglutinantes de fluoruro de polivinilideno (PVDF) con soluciones fuertemente alcalinas. La Granja: Revista de Ciencias de la Vida. Vol. 29(1):17-31. http://doi.org/10.17163/lgr.n29.2019.02.

IDs Orcid:

Leonard Pagliaro: https:/ /orcid.org/0000-0002-3969-2636

Daniel A. Lowy: https:/ / orcid.org/0000-0003-2210-6757 


\section{Introducción}

Politetrafluoroetileno, PTFE, es un material con propiedades únicas, químicamente inerte en medios fuertemente ácidos o alcalinos y en contacto con disolventes orgánicos. También, posee una excelente resistencia al calor y al frío, una llama lenta, y alta adhesividad, y es un buen aislador eléctrico. Sin embargo, el PTFE plantea graves desafíos tecnológicos, ya que es difícil de ser procesado debido a su fibrilación. Se identifica como un polímero que muestra la formación de la microestructura esencial durante el procesamiento en frío, como la extrusión de pasta (Patil y col., 2008; Mitsoulis y Hatzikiriakos, 2009). Dado que la producción de pasta es un paso obligatorio en la fabricación de células galvánicas, en la industria de la batería se prefieren otros aglutinantes que son más rentables y más fáciles de manejar, aumentando el rendimiento y reduciendo el tiempo de inactividad del procesamiento (Vora y Gingras, 2012b; Vora y Gingras, 2012a). Los aglutinantes alternativos que han sido ampliamente utilizados son poli (1, 1-difluoroetileno) o polivinílico (fluoruro del vinilideno), PVDF, y sus copolímeros conocidos bajo el nombre comercial de $\mathrm{Kynar}^{\circledR}(\mathrm{Ar}-$ kema Technical Polymers, 2018).

El polivinílico (fluoruro del vinilideno), PVDF, se considera uno de los polímeros más resistentes químicamente y mecánicamente disponibles comercialmente (Grasselli y Betz, 2005). Por lo tanto, el PVDF es un material muy popular para la fabricación de la membrana, separadores que tienen diferentes usos en varias aplicaciones industriales. Entre las propiedades importantes del PVDF que permiten la formación de membranas es su hidrofobicidad natural, la estabilidad térmica y la inercia química en medios alcalinos (Liu y col., 2011). Debido a estas propiedades, el PVDF sirve como una de los aglutinantes más comunes en la fabricación de baterías. Sin embargo, se demostró que existe una interacción perjudicial de PVDF y de los materiales del cátodo para las baterías del Li-ion. Se comprobó que el contacto de PVDF con $\mathrm{LiCoO}_{2}$ agota la estabilidad de la masa activa. Particularmente en las temperaturas elevadas, la formación de óxido superficial $\left(\mathrm{Co}_{3} \mathrm{O}_{4}\right)$ y la disolución de $\mathrm{Co}$ iones se acelera en los puntos de contacto entre la masa activa y el aglutinante (Markevich, Salitra y Aurbach, 2005). Más recientemente, se han documentado fenómenos similares para las baterías Li-O2, donde el PVDF, un aglutinante de cátodo de uso común, se degrada en presencia de especies de oxígeno reducidas durante la descarga de $\mathrm{Li}-\mathrm{O}_{2}$, cuando hay impurezas presentes (Papp y col., 2017).

Cuando se utiliza en baterías alcalinas, las condiciones básicas pueden rechazar la estabilidad mecánica y térmica de las películas PVDF y pueden causar la degradación de la superficie y las modificaciones estructurales. Dias y McCarthy demostraron que cuando el PVDF original se expone a las soluciones alcalinas fuertes, la superficie del polímero puede ser modificada (Dias y McCarthy, 1984). También se demostró que el procedimiento de deshidrofluoración en la superficie del polímero depende de la temperatura (Shoichet y McCarthy, 1991). Cuando las películas de PVDF se exponen a los medios alcalinos, estas se dañan debido a la formación de poros en la textura del polímero. Se han realizado varios estudios de grabado químico con 9-10 $m o l L^{-1}$ o $20 w t . \%$ de solución de hidróxido sódico en un rango de temperatura de 55 a $85^{\circ} \mathrm{C}$ (Grasselli y Betz, 2005; Komaki y col., 1996; Hashim, Liu y Li, 2011). Mientras que el grabado alcalino a $55-65^{\circ} \mathrm{C}$ produce poros cilíndricos, a $85^{\circ} \mathrm{C}$ se producen poros grabados en forma cónica (Grasselli y Betz, 2005). Incluso las soluciones de $\mathrm{KOH}$ diluidas $(\leq 0,2 \mathrm{M})$ fueron probadas para generar un gran número de poros en la textura del polímero por un proceso de grabado (Samsure y col., 2016). La presencia de un agente oxidante en la solución alcalina amplifica el grabado de PVDF (Grasselli y Betz, 2005). En una investigación del funcionamiento del ciclo de los electrodos del óxido del Fe (III), se compararon cuatro materiales aglutinantes para el uso en la solución alcalina. Se encontró que el PTFE ofrece mejores propiedades que el PVDF (Kitamura y col., 2012).

La deshidrohalogenación de PVDF es un fenómeno conocido y se ha utilizado como método sintético para introducir enlaces dobles en la cadena polimérica, que eventualmente sirvió para injertar otros polímeros. Para permitir la eliminación, se utilizó hidróxido de Tetrametilamonio como base $\left(K_{b}=\right.$ 4,2), (Yan y col., 2016; Zhang, 2015), que está cerca de 4 órdenes de magnitud más débiles que el $\mathrm{KOH}$ utilizado en presente trabajo. Estos estudios de grabado químico sugieren que la exposición de PVDF a soluciones alcalinas deteriora su estabilidad mecánica y térmica. Además, las altas concentraciones alcalinas y altas temperaturas causarían la degradación adicional de PVDF.

La reducción de PVDF en la solución fuertemen- 
te alcalina ocurre probablemente por la pérdida de HF de las cadenas fluoradas del hidrocarburo por medio de la eliminación (Ecuación 1). Los iones del fluoruro se liberaron y forman los enlaces dobles.
Esta reacción puede continuar para formar enlaces dobles adicionales (Ecuación 2). El resultado es una cadena no saturada que contiene un sistema conjugado que absorbe la luz en el rango visible/UV.
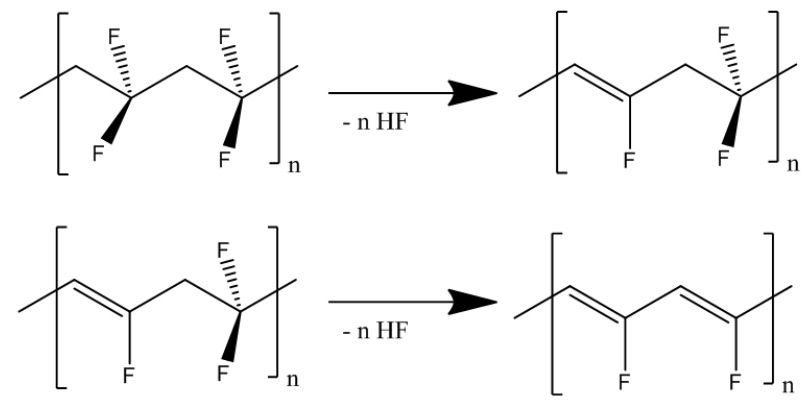

(1)
Con el tiempo, se puede notar un oscurecimiento del polímero (Danks, Slade y Varcoe, 2002; Danks, Slade y Varcoe, 2003; Hinksman, Isaac y Morrissey, 2000). Las cadenas de carbono insaturadas pueden degradarse aún más por oxidación en contacto con los materiales catódicos-activos, $\mathrm{NiOOH}$
(Guiader y Bernard, 2018), $\mathrm{MnO}_{2}$ (Biswal y col., 2015), o $\mathrm{RuO}_{2}$ (Peckerar y col., 2011b; Peckerar y col., 2011a), que sirven como el oxidante. Una posible esquematización del proceso de oxidación se muestra en la Ecuación 3, donde $[O]$ representa al agente oxidante.<smiles>CC(C)(C)C(F)=CC(C)(C)C(F)=CC(C)(C)C(=O)[O-]</smiles>

Todos estos procesos químicos no deseados pueden agotar las propiedades aglutinantes y reducir la vida útil de las células galvánicas. Con el objetivo de evaluar el posible uso del PVDF en el sistema de baterías alcalinas, se evaluó su degradación utilizando una metodología diseñada específicamente para la investigación de la descomposición del aglutinante PVDF en medios alcalinos, tales como la liberación de la concentración de iones $\mathrm{F}$ (expresada en ppm), el cambio de color, la formación de precipitados y los espectros de reflectancia FT-IR.

\section{Materiales y métodos}

\subsection{Productos químicos y materiales}

Los aglutinantes fluorados de PVDF se compraron o fueron regalados por parte de Arkema, Inc. (King of Prussia, Pennsylvania, USA). El Politetrafluoroe- tileno, PTFE, se compró en DuPont, en forma de 60 wt \% suspensión en agua. En la Tabla 1 se observan los nombres comerciales, nombres químicos, números CAS y composiciones de los aglutinantes reportados por el fabricante. Todos los aglutinantes fueron usados sin realizarles ningún cambio. Las soluciones alcalinas acuosas se prepararon disolviendo las pelotillas de $\mathrm{KOH}(\geq 85 \%$, Sigma-Aldrich) en agua desionizada $(18,2 M \Omega \cdot \mathrm{cm}$, sistema de purificación de agua Super-Q ${ }^{\circledR}$ Plus, EMD Millipore). Para la calibración de sondas de fluoruro, se utilizaron 1000 ppm de solución estándar ion-selectiva, fluoruro (Cole-Parmer), mientras que se prepararon $100 \mathrm{ppm}, 10,0 \mathrm{ppm}$, y 1,00 ppm $F^{-}$de soluciones estándares in-House por la dilución serial del 1000 ppm estándar con agua desionizada. El ajustador de fuerza iónico era TISAB 1 (Tampón de ajuste de fuerza iónica total, \#EW-27502-69, kit de solución de doble Unión ISE de fluoruro, Cole-Parmer). 
Tabla 1. Propiedades físicas de los aglutinantes a base de PVDF y PTFE.

\begin{tabular}{|c|c|c|c|}
\hline Nombre comercial & Nombre químico & Número CAS & $\begin{array}{l}\text { Composición/ } \\
\text { Estado físico } \\
\end{array}$ \\
\hline $\begin{array}{c}\text { Kynar Flex }^{\circledR} \\
\text { 2751-00 PVDF }\end{array}$ & $\begin{array}{c}\text { Poli(1,1-difluoroetileno)-co- } \\
\text { hexafluoropropileno (PVDF-HFP) }\end{array}$ & $\begin{array}{c}\text { Copolímero } \\
\# 9011-17-0\end{array}$ & $\begin{array}{l}\text { Relación del monómero } \\
\text { no conocida / Sólido }\end{array}$ \\
\hline $\begin{array}{l}\text { Kynar Ultraflex } \\
\text { RC-10,204 }\end{array}$ & $\begin{array}{c}\text { Poli(1,1-difluoroetileno)-co- } \\
\text { hexafluoropropileno (PVDF-HFP) }\end{array}$ & $\begin{array}{c}\text { Copolímero } \\
\text { PVDF: \#24937-79-9 } \\
\text { HFP: \#116-15-4 }\end{array}$ & $\begin{array}{l}35-55 w t \% \text { copolímero en } \\
\text { agua / suspensión }\end{array}$ \\
\hline $\begin{array}{c}\text { Kynar }^{\mathbb{R}} \\
32 \text { Latex PVDF }\end{array}$ & $\begin{array}{l}\text { Poli(1,1-difluoroetileno) } \\
\text { homopolimero (PVDF) }+C_{7}-C_{13} \\
\text { ácido perfluorocarboxilico, } \\
\text { sal amoníaco (PFAA) }\end{array}$ & $\begin{array}{c}\text { Homopolímero } \\
\text { con aditivos } \\
\text { PVDF: \#24937-79-9 } \\
\text { PFAA: \#72968-38-8 }\end{array}$ & $\begin{array}{l}99 w t \% \text { PVDF } \&<<1 w t . \% \\
\text { PFAA/ en suspensión acuosa }\end{array}$ \\
\hline PTFE TE3859 & $\begin{array}{l}\text { Poli(tetrafluoroetileno), PTFE } \\
\text { Éter de Politilenglicol } \\
\text { (Tergitol }^{\mathbb{R}} \text { TMN) }^{*}\end{array}$ & $\begin{array}{c}\text { PTFA: \#9002-84-0 } \\
\text { Tergitol: \#60828-78-6 }\end{array}$ & $\begin{array}{c}45-65 w t \% \text { polímero, } 1-10 w t \% \\
\text { Tergitol en } 25-50 w t \% \\
\text { agua / suspensión }\end{array}$ \\
\hline
\end{tabular}

* Tergitol TMN actúa como surfactante para el mantenimiento de PTFE en suspensión acuosa.

\subsection{Métodos}

\subsubsection{Muestras utilizadas para la medición de fluo- ruro}

Se realizaron experimentos con 4 aglutinantes (recibidos como sólidos o dispersiones acuosas), los cuales estuvieron expuestos a una solución acuosa de $32 w t \%$ de $\mathrm{KOH}(p H>14)$, a $60^{\circ} \mathrm{C}$, para aumentar la duración. La liberación de iones $F^{-}$se monitoreó con el tiempo mediante un electrodo de combinación de fluoruro (para más detalles ver sección 2.2.4). Luego, se construyeron células galvánicas utilizando 4 pastas de cátodos diferentes, conteniendo cada uno de los aglutinantes enumerados en la Tabla 1. Cada pasta de cátodo se llevó a un colector, y luego se enrolló con un separador de batería, y se sumergió en solución acuosa $32 w t \%$ de solución $\mathrm{KOH}$. Este enfoque imita el uso de aglutinantes en las células galvánicas en la vida real. Una película de olefina sulfonada, Freudenberg FV-4304, sirvió como separador (Freudenberg Performance Materials, LP, Durham, Carolina del norte). Cada rollo estaba atado con cinta CURAPROX PTFE resistente al desgarro (código DF820, Curaden AG, Kriens, Suiza), y luego, estos cátodos se sumergieron de manera individual en $32 w t \%$ solución acuosa de $\mathrm{KOH}$, en un vial de polipropileno separado para duraciones cronometradas.

\subsubsection{Preparación de la muestra}

La preparación de la muestra implicó tres pasos: (i) se filtró la parte precipitada del aglutinante (II) la muestra se enfrió en el baño de hielo con el fin de evitar posibles daños causados por el calor liberado sobre la neutralización, en el siguiente paso; (III) la muestra fue llevada a pH 5,5-6,5 mediante neutralización con ácido acético glacial. Según nuestro protocolo, se añadió 14,7 g de agente de amortiguación de ácido acético glacial a 22,4 g solución filtrada, de tal manera que la muestra se ajustó a un de pH 6,5 $\pm 0,2$, que es el rango ideal para la determinación de iones de fluoruro.

\subsubsection{Prevención de interferencias en la respuesta de la sonda selectiva $F$}

Se tomó precaución en no contaminar las muestras con aniones interferentes, incluyendo haluros $\left(\mathrm{Cl}^{-}\right.$, $\left.\mathrm{Br}^{-}, \mathrm{I}^{-}\right)$, nitrato, sulfato, etc. Para evitar la contaminación de la muestra, se evitó el uso de envases de vidrio durante todo el proceso de preparación de la muestra (descrito en la sección 2.2.4). Todas las muestras se diluyeron, según fue necesario, en matraces volumétricos de polipropileno (matraces volumétricos de plástico Corning PP, $100 \mathrm{~mL}, \pm 0,20$ $\mathrm{mL} \mathrm{a} 20^{\circ} \mathrm{C}$ ), y todas las mediciones de concentración de iones $F^{-}$se realizaron en vasos de polipropileno (Cole-Parmer Tri-Corner Beaker, PP, 100 mL).

\subsubsection{Monitoreo mediante potenciómétro de la con- centración de fluoruro}

Se determinó la concentración de $F^{-}$en las muestras filtradas mediante el uso de un electrodo epoxi de combinación de iones de fluoruro (CAT. No. 27504-14, Cole-Parmer), con un rango de medida de 
$1.0 \mathrm{~mol} L^{-1}$ to $1.0 \mu \mathrm{mol} L^{-1}$, unido a un medidor modelo Orion Star A211 $\mathrm{pH} / \mathrm{mV}$ (Thermo Scientific). Las mediciones se realizaron con un $\mathrm{pH} \sim 6,5$.

En cada día de mediciones, la sonda selectiva $F^{-}$ fue calibrada con 4 soluciones estándares. La curva de calibración de la sonda fue lineal sobre 3 órdenes de magnitud de la concentración $F^{-}$(1-1000 ppm), con una pendiente ligeramente sobre Nernstian de $62,1 \mathrm{mV} /$ (década de concentración $F^{-}$), y cerca del coeficiente de correlación teórica de $R^{2}=0,9998$. La sonda fue recalibrada por calibración de 4 puntos todos los días. Además, antes de cada medición, se comprobó de nuevo el electrodo selectivo de la pendiente de $F^{-}$por medio de la calibración de 2 puntos, con dos soluciones estándares conteniendo 10 y $100 \mathrm{ppm} F^{-}$, respectivamente. La fuerza iónica de cada solución se ajustó al valor constante, añadiendo TISAB 1 (tampón de Ajustador de fuerza iónica total, \#EW - 27502 - 69, kit de solución de doble Unión de fluoruro ISE, Cole-Parmer). Esta operación es impuesta por la necesidad de fuerza iónica alta y por la constante en relación con la concentración de iones de $F^{-}$, de modo que el coeficiente de actividad, $\gamma$, es constante; por la tanto, la actividad de fluoruro es directamente proporcional a la concentración de flúor.

Dado que las dos normas de calibración consecutivas abarcan un decenio de concentración, la diferencia del potencial registrado en $\mathrm{mV}$ corresponde a la pendiente de la sonda ( $\mathrm{mV} /$ década).

\subsubsection{Cambio de color de los aglutinantes}

El cambio de color de los aglutinantes en función del tiempo de exposición a la solución alcalina fue monitoreado en la solución clara, después de eliminar los sólidos por filtración de gravedad. Las fotografías se tomaron periódicamente y se compararon visualmente para evaluar los cambios de color. También, se registró la formación de precipitados.

\subsubsection{Espectros FT-IR}

Se grabaron los espectros FT-IR de los polímeros PVDF antes y después de haber sido expuestos a la solución de hidróxido concentrado. Después de la exposición, el sólido fue recogido por la filtración de la gravedad, se enjuagó 7 veces con el DIW para quitar cualquier rastro de $\mathrm{KOH}$, y después se secó en el aire, a temperatura ambiente durante $48 h$. Los espectros FT-IR de las muestras de polímero se registraron en modo de reflectancia; como controles se utilizaron muestras del polímero idéntico que no había sido expuestas a los medios alcalinos. Los espectros se grabaron en un instrumento modelo Nexus 870 FT-IR ESP, fabricado por Thermo Nicolet, en el rango espectral de 4000 a $400 \mathrm{~cm}^{-1}$. Como se muestra en el apéndice, todos los espectros se registraron como absorbancia versus longitud de onda.

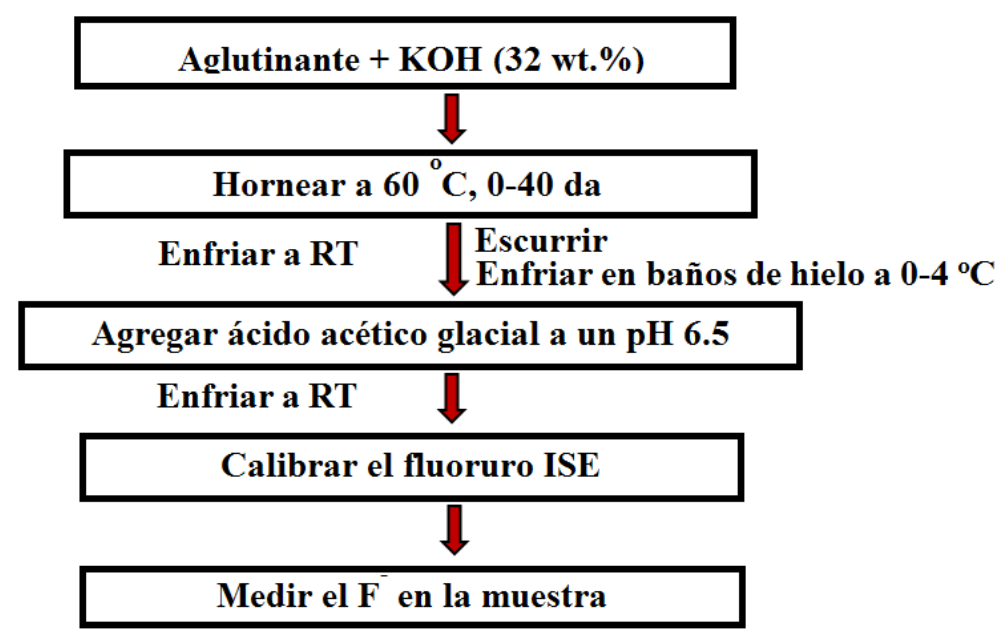

Figura 1. Esquema de la preparación de la muestra y del procedimiento analítico. 


\section{Análisis y resultados}

Un detalle importante de la metodología implementada fue operar la sonda selectiva en solución ligeramente ácida, instrucciones impuestas por el fabricante (Cole-Parmer Company Fluoride Ion Electrodes, 2018). Como se describió, las soluciones de muestra utilizadas en este estudio fueron muy básicas $(p H>14)$. Por lo tanto, antes de cualquier medida potenciométrica, la preparación de la muestra fue necesaria, de acuerdo con la metodología descrita en la sección 2.2.2. En la Figura 1 se muestra un esquema del procedimiento analítico y de pre- paración de la muestra.

El estado físico de un aglutinante fue sólido, mientras que los otros 3 fueron obtenidos en forma de suspensiones acuosas (ver Tabla 1). La masa de cada muestra de aglutinante tomada para estas pruebas se calculó de tal manera que contenía aproximadamente $1 \mathrm{~g}$ de material sólido. Por lo tanto, la concentración de iones $F^{-}$medida (expresada en ppm), liberada de la muestra de aglutinante, es paralela a la pérdida masiva de aglutinante (en \% de masa), causada por la liberación de iones $F^{-}$. El aglutinante PTFE sumergido en la misma solución fuertemente alcalina sirvió como el experimento del control.

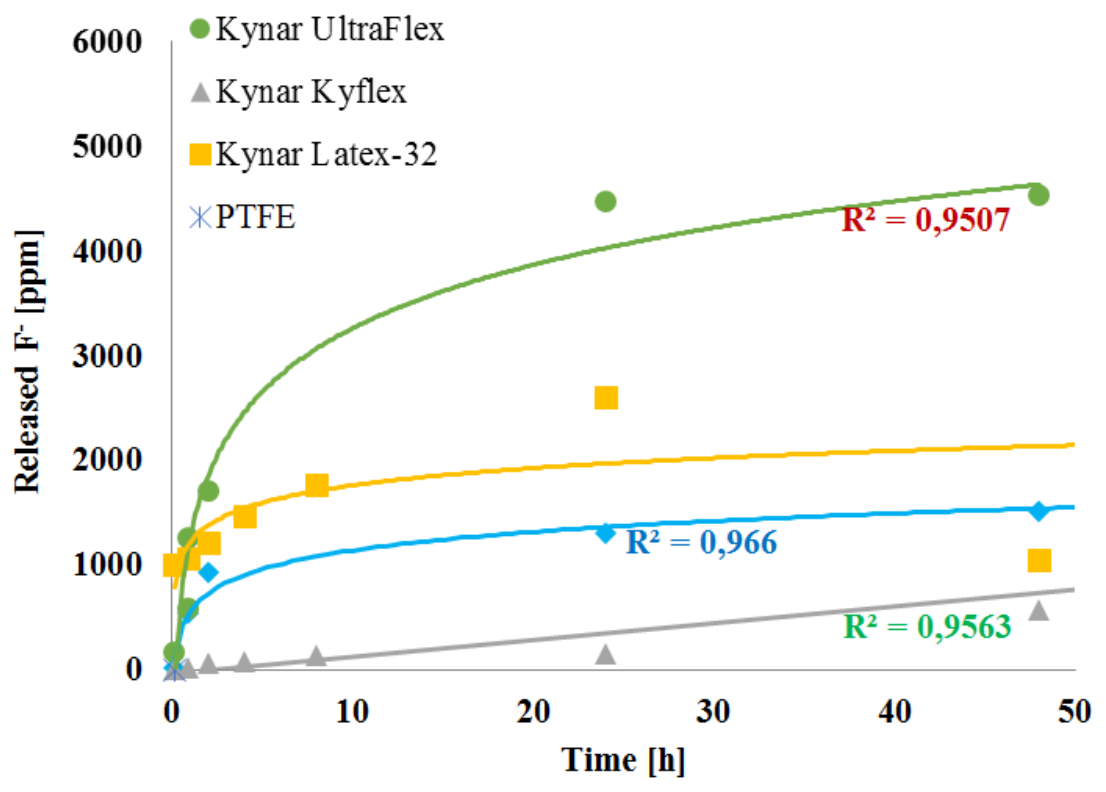

Figura 2. Liberación de iones de fluoruro durante 2 días a partir de 4 aglutinantes fluorados diferentes al sumergirse en $32 w t \%$ solución de $\mathrm{KOH}$, y mantenido en $60^{\circ} \mathrm{C}$. A mayor concentración de $F^{-}$(en ppm) medida sobre $48 \mathrm{~h}$, mayor el grado de degradación de la aglomerante. El control de PTFE liberó concentraciones insignificantes de $F^{-}$(ver marca en la parte inferior del gráfico).

Las curvas en la Figura 2 ilustran la liberación de iones $F^{-}$de cada aglutinante con el tiempo. La pérdida de fluoruro agota las propiedades aglutinantes, causando la degradación del aglutinante. Los datos para PTFE están cerca de 0 ppm de $F^{-}$liberado; por lo tanto, se alinean a lo largo del eje $\mathrm{x} y$ no se pueden ver en el gráfico. Fuera de los aglutinantes puros expuestos a $32 w t \%$ solución de $\mathrm{KOH}$, Kynar ultra Flex se degrada a un ritmo más rápido; dado que es una suspensión, el polímero fluorado contenido en el aglutinante tiene el área de con- tacto más grande con el electrólito. Por el contrario, Kynar Flex, que es sólido, muestra una degradación mucho más lenta (ver triángulos verdes en la Figura 2).

A continuación, se imitó el entorno real del aglutinante, cuando fue encontrado en la investigación y el desarrollo de baterías alcalinas. Cada aglutinante se mezcló con la pasta de cátodo y luego la pasta se aplicó al colector de corriente. El contenido sólido de aglutinante de cada pasta dentro del cátodo fue expresado como wt \% según se indica en la Ta- 
bla 2. Las muestras de cátodo se pesaron y se enrollaron con el separador de poliolefina sulfonada (Freudenberg FV-4304). Los rollos obtenidos se ataron con cinta de PTFE y se sumergieron en una solución de $\mathrm{KOH}$ al $32 w t \%$ en peso, cada rollo en un vial de plástico separado. Luego, todos los viales se colocaron en una cámara ambiental y se mantuvieron a $60^{\circ} \mathrm{C}$. De nuevo, el control del experimento fue la tasa de degradación del aglutinante de PTFE. Los resultados del monitoreo de la liberación de fluoruro se muestran en la Figura 3.

Tabla 2. Contenido de aglutinante y acondicionamiento de cátodos.

\begin{tabular}{cccc}
\hline Aglutinante & $\begin{array}{c}\text { Contenido sólido del } \\
\text { aglutinante en el cátodo }\end{array}$ & Secado en aire & Estabilizado \\
\hline Kynar Flex & $2,44 w t \%$ & N/A & $150^{\circ} \mathrm{C}, 15 \mathrm{~min}$ \\
\hline Kynar Ultra Flex & $3,36 w t \%$ & $60^{\circ} \mathrm{C}, 1 \mathrm{~h}$ & $95^{\circ} \mathrm{C}, 15 \mathrm{~min}$ \\
\hline PVDF Latex-32 & $4,24 w t \%$ & $60^{\circ} \mathrm{C}, 1 \mathrm{~h}$ & $150^{\circ} \mathrm{C}, 15 \mathrm{~min}$ \\
\hline PTFE & $0,988 w t \%$ & $60^{\circ} \mathrm{C}, 1 \mathrm{~h}$ & $\mathrm{~N} / \mathrm{A}$ \\
\hline
\end{tabular}

Para las celdas de cátodo investigadas, se encontró que, en 1 semana a $60^{\circ} \mathrm{C}$, el aglutinante Kynar ultra Flex se degradó a la tasa más alta, seguida de PVDF latex-32 y Kynar Flex. El experimento del control con PTFE demostró degradar a un grado insignificante. El cambio de color (oscurecimiento de la solución de la muestra) o la formación de precipi- tados (causada por el desprendimiento del material activo de cátodo del colector) son similares a los degradantes evaluados por el aumento de la concentración de fluoruro. Los espectros FT-IR revelan la desaparición de los enlaces $C-F$ como resultado de la degradación de PVDF, lo que provocó el aumento de la desaturación de la cadena de carbono.

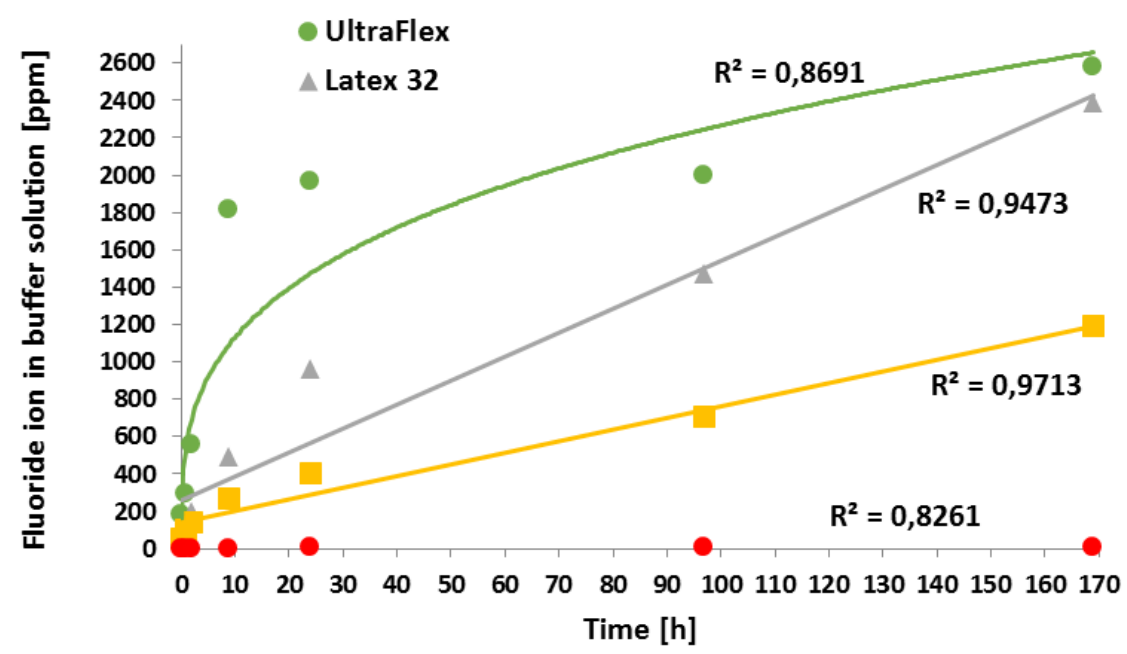

Figura 3. Degradación de los aglutinantes de las celdas a lo largo del tiempo, cuando se exponen en solución de KOH a $32 w t \%$. Se realizaron pruebas en aglutinantes incorporados en medias celdas, y la concentración de $F^{-}$(en ppm) se monitoreó durante 1 semana. El control de PTFE liberó concentraciones insignificantes de $F^{-}$(ver círculos rojos en la parte inferior del gráfico) 


\section{Discusión y conclusiones}

La liberación de fluoruro de diferentes aglutinantes muestra una tendencia similar para los aglutinantes libres (no incorporada en la pasta cátodo, mostrada en la Figura 2), y para los aglutinantes presentes en las celdas (Figura 3). La pérdida de fluoruro de los aglutinantes se eleva en el orden mostrado a continuación, que también corresponde a la magnitud de la degradación química de los aglutinantes:

PTFE $\ll$ Kynar Flex $<$ PVDF Latex-32 « Kynar Ultra Flex

En las Figuras 4 y 5 se muestra la prueba visual de la degradación del aglutinante:
- Con el tiempo, el latex-32 exhibe un cambio de color (oscurecimiento de la solución), Figura 4 .

- PTFE no muestra ningún signo de degradación (ya sea cambio de color o formación de precipitados), Figura 5.

Por último, en la Figura 6 se comparan los cátodos con Kynar ultra Flex (el más estable de los aglutinantes Kynar investigados) y los cátodos preparados con PTFE (control). Las imágenes se tomaron después de 168 h de exposición a la solución $\mathrm{KOH}$ a $32 w t \%$ (sirviendo como electrolito típico en celdas alcalinas). La Tabla 2 revela más vertimiento del cátodo obtenido con Kynar ultra Flex que con PTFE.

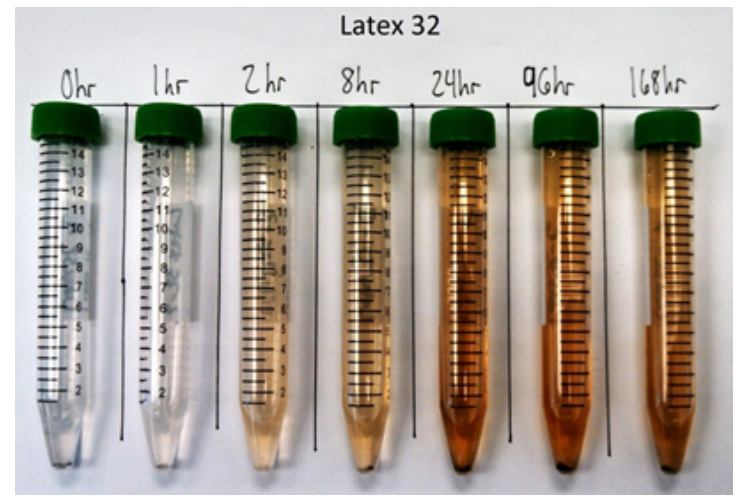

Figura 4. Fotografía que muestra el cambio de color de la solución de $\mathrm{KOH}$ a $32 w t \%$ y el precipitado formado a lo largo del tiempo para PVDF latex-32; el oscurecimiento corresponde al número cada vez mayor de enlaces dobles sobre las cadenas de carbono, debido a la pérdida de fluoruro; el precipitado consiste en partículas que se derraman del cátodo y se colocan en la parte inferior del vial.

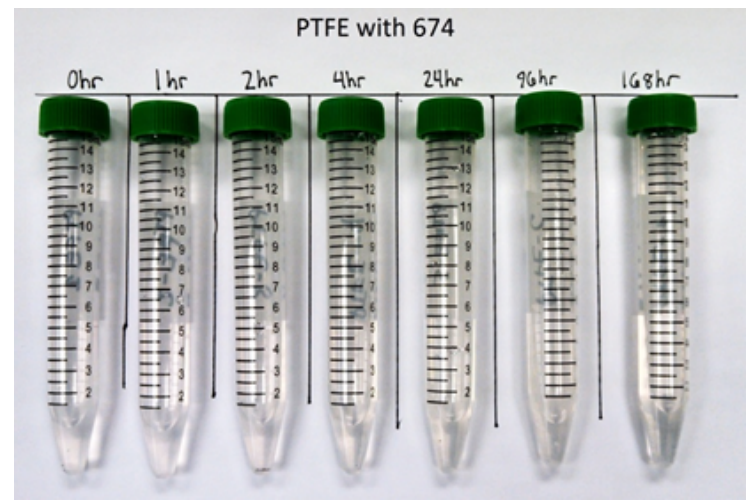

Figura 5. Fotografía que muestra el cambio de color de la solución $\mathrm{KOH}$ a $32 w t \%$ y precipitado formado a lo largo del tiempo para PTFE, utilizado como control; la solución fuertemente alcalina permanece clara y no hay precipitado notable. 
El apéndice indica espectros FT-IR sobrepuestos; se analizó cada aglutinante después de haber sido expuesto a solución de $\mathrm{KOH}$ en $32 w t \%$, y luego superpuesto con el espectro del aglutinante inicial sin tratar. Las asignaciones de bandas espectrales se basan en Robinson, (1974) y en otros autores (Bhullar, 2014; Smith, 2011; Stuart, 2004; Ningrum y Kusumawati, 2016; Perusich, 2000). A excepción de PTFE, cada aglutinante muestra un cambio en sus cadenas $C-F$, es decir, la absorción de este enlace obtiene el más agotado o el menos agotado, demostrando que parte de las cadenas $C-F$ son descompuestas por el electrolito fuertemente alcalino. Los espectros de la Figura 1A revelan un cambio significativo en la composición del aglutinante. Sobre la inmersión en la solución alcalina concentrada, la banda de absorción de estiramiento $>\mathrm{CF}_{2}$ casi desaparece, mientras que las bandas de absorción adicionales aparecen en la región $1575-1660 \mathrm{~cm}^{-1}$, que se puede asignar a la formación de enlaces dobles $-C=C-$. Algunos de estos enlaces $\pi$ pueden someterse a reacciones de adición de moléculas de agua, y formar grupos carbonilos que se absorben a $1700 \mathrm{~cm}^{-1}$ (ver Figura 1A). Un sistema de doble enlace conjugado $(-C=C-C=C-C=C-$ ) representa el color marrón anaranjado evaluado para varias de las soluciones de aglutinante (ver Figura 5). La formación del precipitado corresponde al vertimiento de los materiales cátodo-activos de la espuma del níquel. La magnitud de la degradación química de los aglutinantes aumenta en el orden:

\section{PTFE $\ll$ Kynar Flex $<$ PVDF Latex-32 « Kynar Ultra Flex}

Se estima que la metodología reportada en esta investigación permitirá monitorear la liberación de fluoruro de cualquier aglutinante que contenga fluoruro cuando esté presente en las celdas completas. Por lo tanto, estas investigaciones brindan a los expertos información de calidad sobre la estabilidad esperada de los aglutinantes antes de ser probados en celdas galvánica ensambladas, ahorrando tiempo y esfuerzo, y agilizando la investigación y el desarrollo de la batería.

\section{Kynar Ultraflex $168 \mathrm{hr}$ half cell}

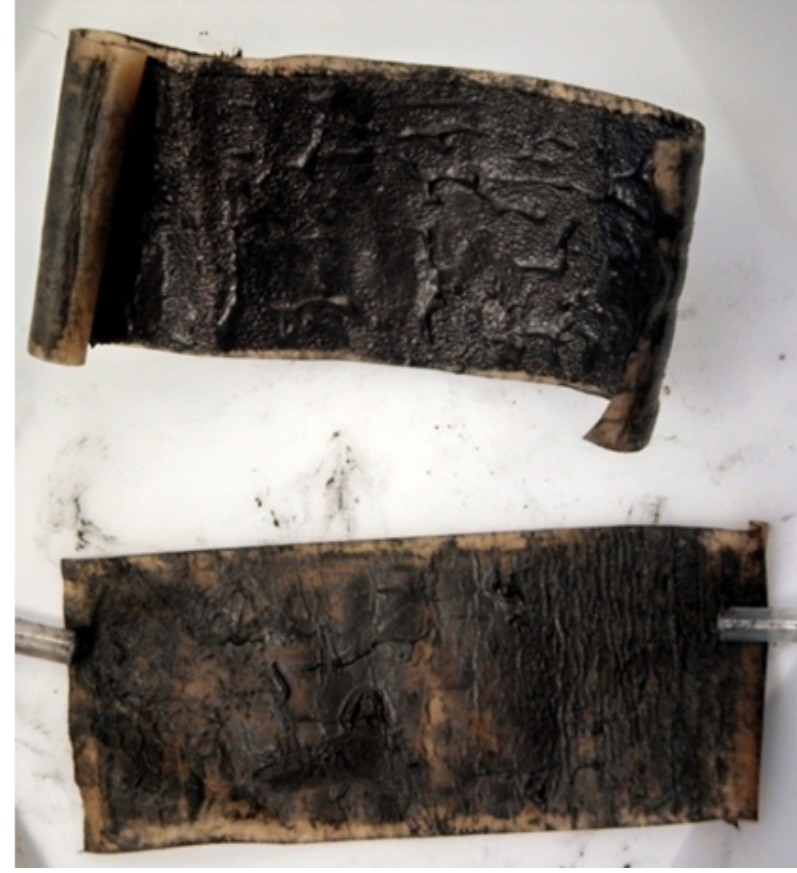

\section{PTFE with $674168 \mathrm{hr}$ half cell}

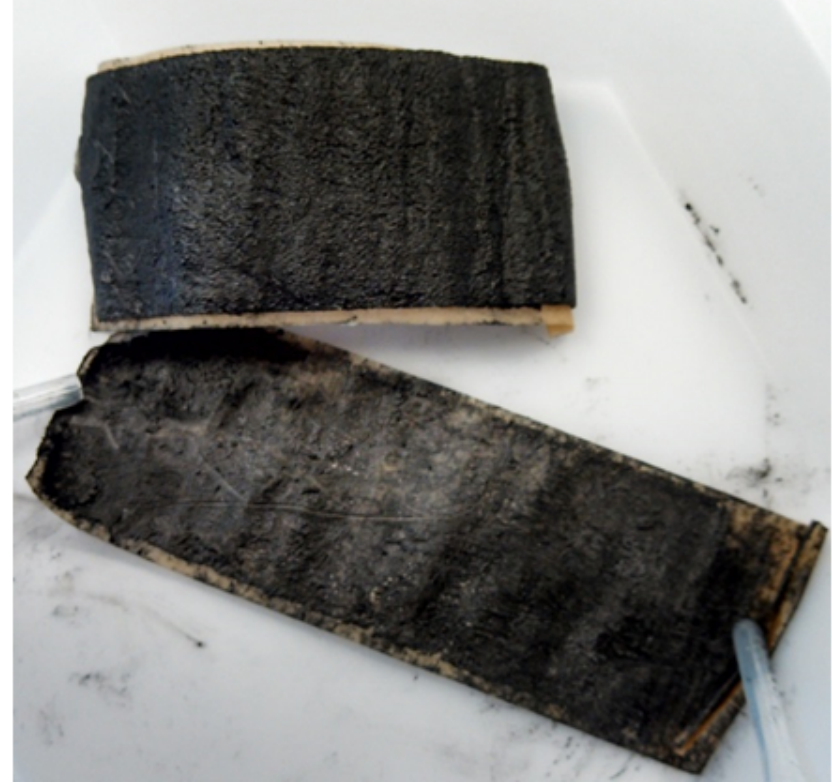

Figura 6. Más desprendimiento del cátodo expuesto a la acción de la solución de KOH a $32 w t \%$ (el electrólito en baterías alcalinas) se puede observar para Kynar ultra Flex y no para PTFE (en cada fotografía la imagen superior es la espuma de NI, mientras que la parte inferior es el separador). 


\section{Agradecimientos}

Los autores agradecen al Dr. Jean-François Audebert, CEO y Presidente de FlexEl, LLC, por la valiosa colaboración y el estímulo permanente brindado para llevar a cabo este estudio y difundir sus resultados. De igual forma, agradecen a la La Sra. Mahsa Dornajafi por proporcionar su asistencia técnica, lo que permitió acelerar la labor de la presente investigación.

\section{Referencias}

Arkema Technical Polymers (2018). Materials Database. Arkema Technical Polymers.

Bhullar S. K. ; Bedeloglu, Ayse ; Martin B. G. (2014). «Characterization and Auxetic effect of Polytetrafluoroethylene Tubular structure». En: International journal of Advanced Science and Engineering 1.2, 8-13. Oline:/urlhttps://bit.ly/2GB4sTu.

Biswal, A. y col. (2015). «Electrodeposition of manganese dioxide: effect of quaternary amines». En: Journal of Solid State Electrochemistry 17.5, 1349-1356. Online:/urlhttps://bit.ly/2BK1HOj.

Cole-Parmer Company Fluoride Ion Electrodes (2018). Instruction Manual. Cole-Parmer Company. Online:https://bit.ly/2EnZB5V.

Danks, T. N., R. C. T. Slade y J. R. Varcoe (2003). "Alkaline anion-exchange radiationgrafted membranes for possible electrochemical application in fuel cells». En: Journal of Materials Chemistry 13.4, 712-721. Online:/urlhttps://bit.ly/2GRYOfb.

Danks, T. N., R. T. Slade y J. R. Varcoe (2002). «Comparison of PVDF-and FEP-based radiationgrafted alkaline anion-exchange membranes for use in low temperature portable DMFCs». En: Journal of Materials Chemistry 12.12, 3371-3373. Online:/urlhttps://rsc.li/2XcNakX.

Dias, A. J. y T. J. McCarthy (1984). «Synthesis of a two-dimensional array of organic functional groups: surface-selective modification of poly (vinylidene fluoride)». En: Macromolecules 17.12, 2529-2531. Online:/urlhttps:/ / bit.ly/2X8DEiC.

Grasselli, M. y N. Betz (2005). «Making porous membranes by chemical etching of heavy-ion tracks in $\beta$-PVDF films». En: Nuclear Instruments and Methods in Physics Research Section B: Beam Interactions with Materials and Atoms 236.1-4, 501-507. Online:/urlhttps://bit.ly/2NgrH5M.
Guiader, O. y P. Bernard (2018). «Understanding of $\mathrm{Ni}(\mathrm{OH})_{2} / \mathrm{NiOOH}$ Irreversible Phase Transformations: $\mathrm{Ni}_{2} \mathrm{O}_{3} \mathrm{H}$ Impact on Alkaline Batteries». En: Journal of The Electrochemical Society 165.2, A396-A406. Online:/url.

Hashim, N. A., Y. Liu y K. Li (2011). «Preparation of PVDF hollow fiber membranes using $\mathrm{SiO}_{2}$ particles: the effect of acid and alkali treatment on the membrane performances». En: Industrial \& Engineering Chemistry Research 50.5, 3035-3040. Online:/urlhttps://bit.ly/2EiVNCM.

Hinksman, P., D. H. Isaac y P. Morrissey (2000). «Environmental stress cracking of poly (vinylidene fluoride) and welds in alkaline solutions». En: Polymer Degradation and Stability 68.2, 299-305. Online:/urlhttps://bit.ly/2VaAh9d.

Kitamura, H. y col. (2012). "Effect of binder materials on cycling performance of $\mathrm{Fe}_{2} \mathrm{O}_{3}$ electrodes in alkaline solution». En: Journal of Power Sources 208, 391-396. Online:/urlhttps://bit.ly/2SNrnle.

Komaki, Y. y col. (1996). "Radicals in heavy ion-irradiated polyvinylidene fluoride». En: Radiation Measurements 26.1, 123-129. Online:/https://bit.ly/2T3vnx3.

Liu, F. y col. (2011). "Progress in the production and modification of PVDF membranes». En: Journal of membrane science 375.1-2. Online:/urlhttps:/ / bit.ly/2SdM3Oa, págs. 1-27.

Markevich, E., G. Salitra y D. Aurbach (2005). "Influence of the PVdF binder on the stability of $\mathrm{LiCoO}_{2}$ electrodes». En: Electrochemistry communications 7.12, 1298-1304. Online:/urlhttps://bit.ly/2GRNM9G.

Mitsoulis, E. y S. G. Hatzikiriakos (2009). «Steady flow simulations of compressible PTFE paste extrusion under severe wall slip». En: Journal of Non-Newtonian Fluid Mechanics 157.1-2, 26-33. Online:/urlhttps://bit.ly/2DVviCh.

Ningrum, Ruwanti Dewi Cahya y Nita Kusumawati (2016). "Development and Characterization of Polysulfone/Polyvinylidene Flouride Blend Membrane Induced by Delayed Liquid-Liquid Demixing». En: International Journal on Advanced Science, Engineering and Information Technology 6.5, 716-722. Online:/urlhttps://bit.ly/2NfBdX9.

Papp, Joseph K. y col. (2017). «Poly (vinylidene fluoride)(PVDF) binder degradation in $\mathrm{Li}-\mathrm{O}_{2}$ batteries: a consideration for the characterization of lithium superoxide». En: The journal of 
physical chemistry letters 8.6, 1169-1174. Online:/https:/ / bit.ly/2T6Q9vZ.

Patil, P. D. y col. (2008). "Viscoelastic flow simulation of polytetrafluoroethylene (PTFE) paste extrusion». En: Journal of NonNewtonian Fluid Mechanics 153.1, 25-33. Online:https:/ / bit.ly/2Xf7bHF.

Peckerar, Martin y col. (2011a). "A novel high energy density flexible galvanic cell». En: Energy $\mathcal{E}$ Environmental Science 4.5, 1807-1812. Online:/urlhttps:/ /rsc.li/2EmhEto.

Peckerar, Martin y col. (2011b). «Fabrication of flexible ultracapacitor/galvanic cell hybrids using advanced nanoparticle coating technology». En: Journal of Vacuum Science \& Technology B, Nanotechnology and Microelectronics: Materials, Processing, Measurement, and Phenomena 29.1, 011008. Online:/urlhttps://bit.ly/2BKnPFM.

Perkin-Elmer (2018). Polymer Identification Using Mid Infrared Spectroscopy. Perkin-Elmer.

Perusich, Stephen A (2000). "Fourier transform infrared spectroscopy of perfluorocarboxylate polymers». En: Macromolecules 33.9, 3431-3440. Online:/urlhttps://bit.ly/2GS712Q.

Robinson, James W. (1974). Handbook of Spectroscopy: Volume I. CRC press.

Samsure, Nurul Alwani y col. (2016). «Alkaline etching treatment of PVDF membrane for water filtration». En: RSC Advances 6.26, 22153-22160. Online:/urlhttps://rsc.li/2tvUQRJ.

Shoichet, M. S. y T. J. McCarthy (1991). «Convenient syntheses of carboxylic acid functionalized fluoropolymer surfaces». En: Macromolecules 24, 982-986. Online:/urlhttps://bit.ly/2ScTt3Z.

Smith, Brian C (2011). Fundamentals of Fourier transform infrared spectroscopy. CRC press.

Stuart, B. H. (2004). Infrared Spectroscopy: Fundamentals and Applications. 1st. Chichester (West Sussex, England): John Wiley \& Sons, Ltd.

Vora, V. y J. Gingras (2012a). «PVDF Fluoropolymer process aids: For best-in-class performance,» en: Modern Plastics $\mathcal{E}$ Polymers september, págs. 71-72.

- (2012b). «PVDF fluoropolymer process aids: Increasing throughput, reducing downtime». En: Modern Plastics \& Polymers, january, págs. 190-192.

Yan, Kai-bo y col. (2016). «Preparation and Characterization of Oil/Water Separation Membranes via Grafting Methyl Methacrylate onto Poly (vinylidene fluoride». En: Acta Polymerica Sinica 5, págs. 659-666.

Zhang Jia-Han ; Guo, Gui-Bao ; AN Sheng-Li; Hao Yan; Zhang Dong; Yan Kai-Bo (2015). «Synthesis and properties of proton exchange membranes via single-step grafting PSBMA onto PVDF modified by TMAH». En: Acta Physico-Chimica Sinica 31.10, 1905-1913. Online:/urlhttps://bit.ly/2TW9BIT. 


\section{Apéndice}

\section{A Estudios FT-IR sobre aglutinan- tes iniciales y degradados}

Para facilitar la visión general, cada banda IR ha sido marcada con la vibración molecular a la que corresponde. Los espectros revelan un cambio signi- ficativo en la composición del aglutinante. En relación al tratamiento de $\mathrm{KOH}$ la banda de absorción de estiramiento $>C F_{2}$ casi desaparece, mientras que las bandas de absorción adicionales aparecen en la región $1575-1660 \mathrm{~cm}^{-1}$, que corresponde a la presencia de enlaces dobles conjugados. Algunos de estos pueden agregar una molécula de agua y formar grupos carbonilos que absorben a $1700 \mathrm{~cm}^{-1}$ (ver las líneas en la figura 1A).

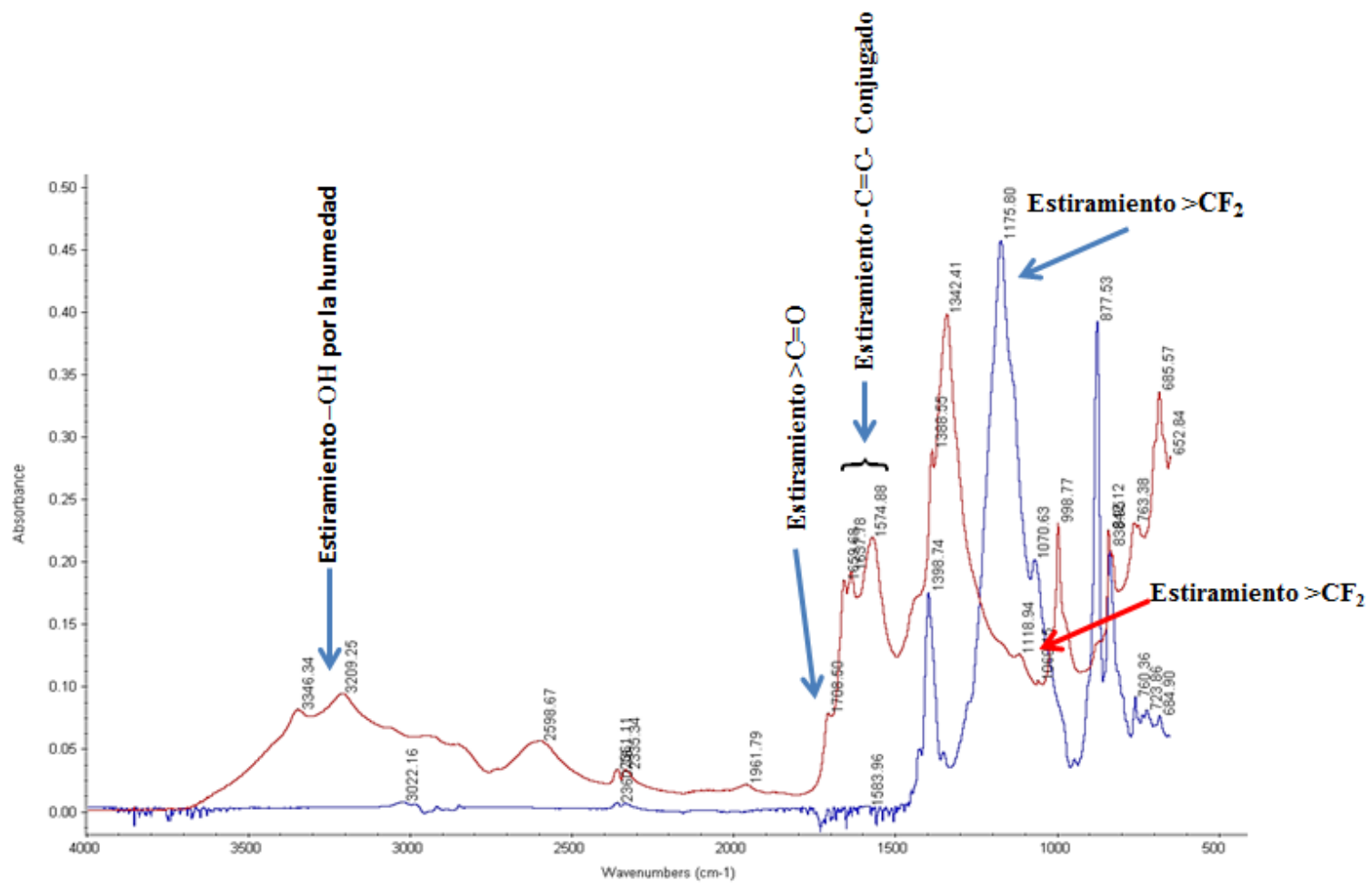

Figura 1. A. Superposición de espectros IR para Kynar UltraFlex: azul - aglutinante inicial, rojo - después de 48 h de exposición a solución $\mathrm{KOH}$ a $32 w t \%$, a $60^{\circ} \mathrm{C}$. 


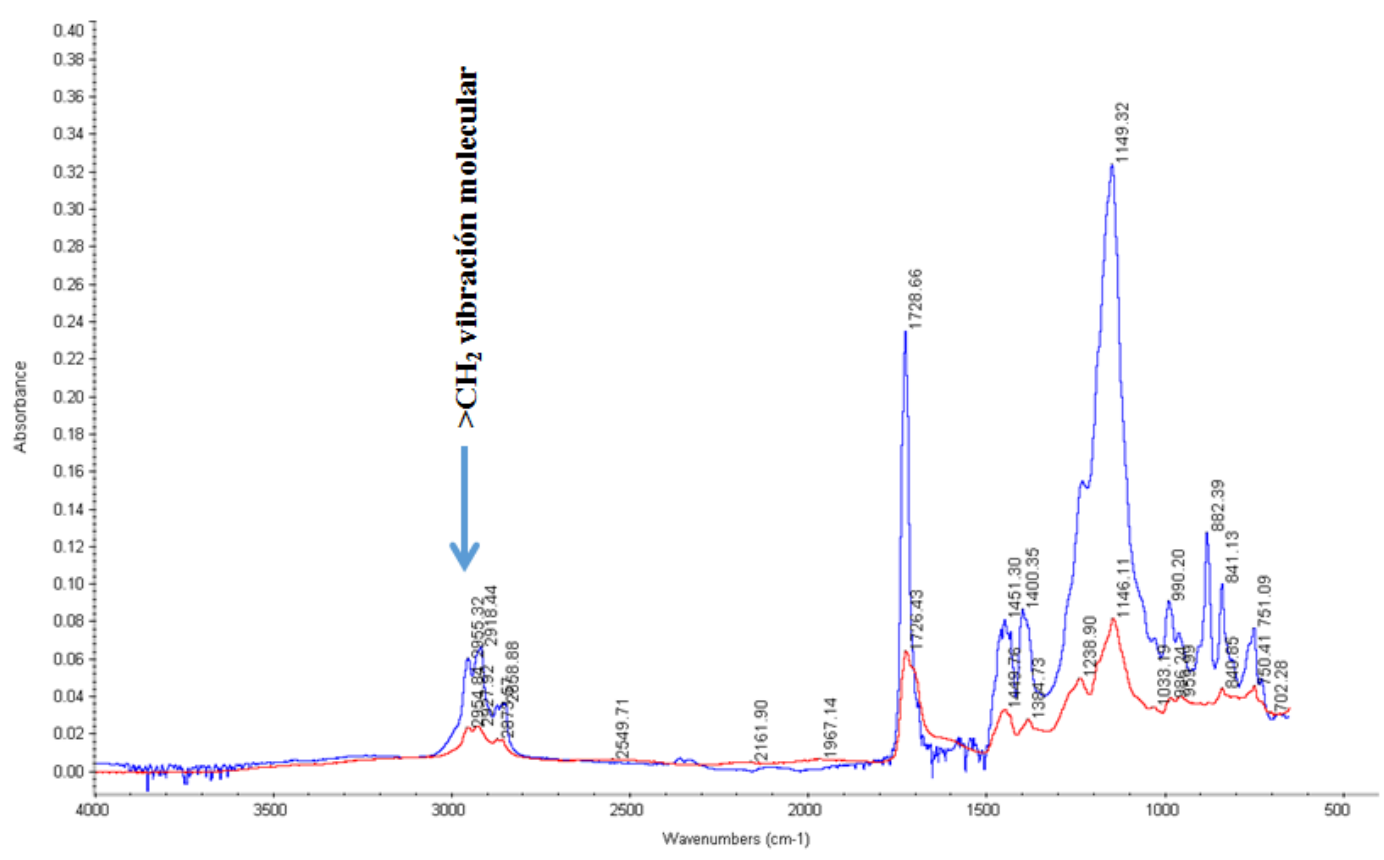

Figura 2. A. Superposición de espectros IR para Kynar Kynar Flex: azul - aglutinante inicial, rojo - después de 48 h exposición a solución de $\mathrm{KOH}$ a $32 w t \%$, a $60^{\circ} \mathrm{C}$. No se puede evaluar ningún cambio relevante en la estructura del polímero (las alturas máximas difieren debido a la cantidad de la muestra).

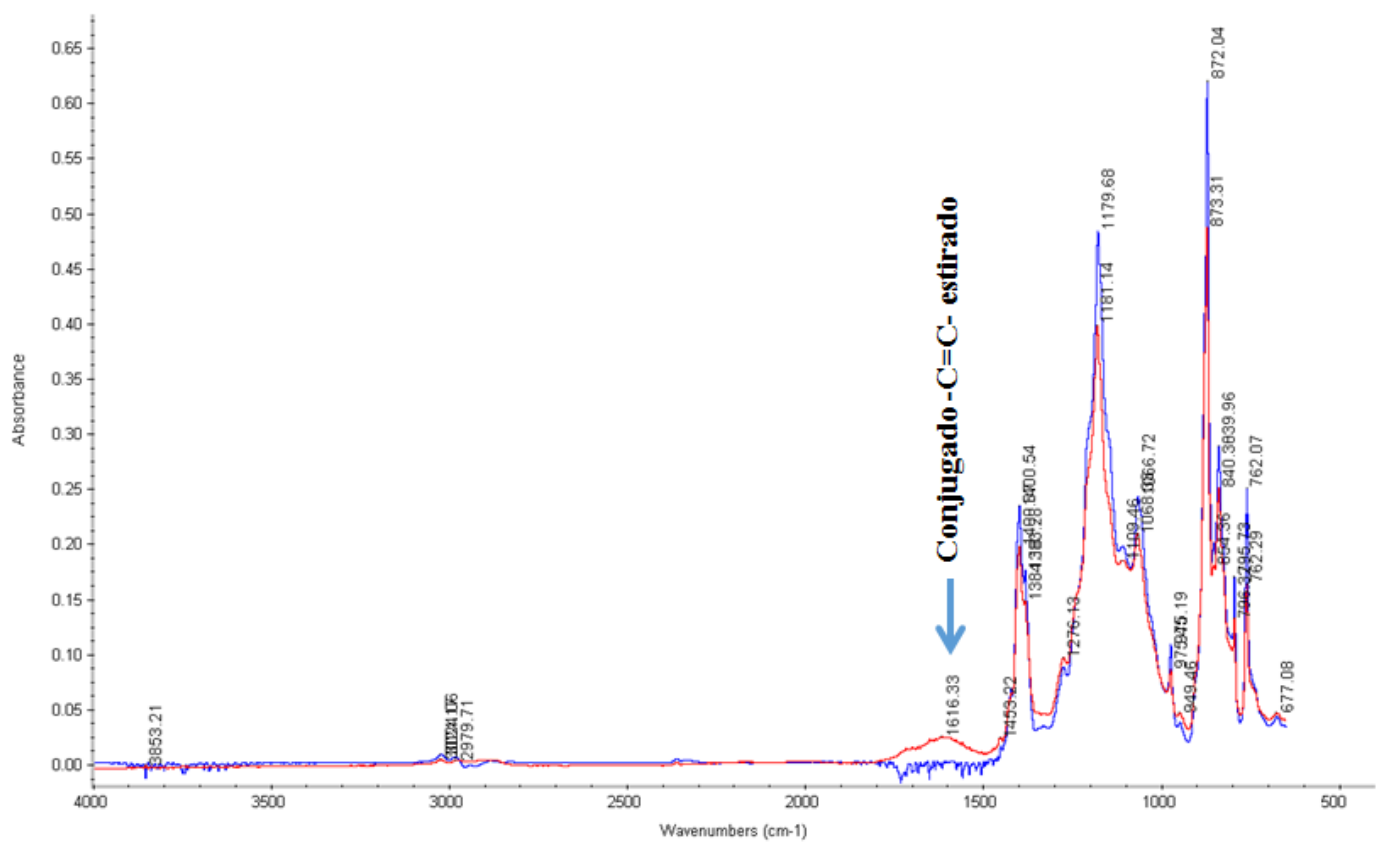

Figura 3. A. Superposición de espectros IR para PVDF latex-32: azul - aglutinante inicial, rojo - después de 48 h exposición solución KOH a 32wt \%. En cuanto a Kynar Kynar Flex (Figura 2), la banda de absorción de los enlaces dobles conjugados aparece después de exponer el aglutinante al electrolito alcalino durante 1 semana a $60^{\circ} \mathrm{C}$. Sin embargo, el cambio en la estructura del polímero no parece ser notorio. 


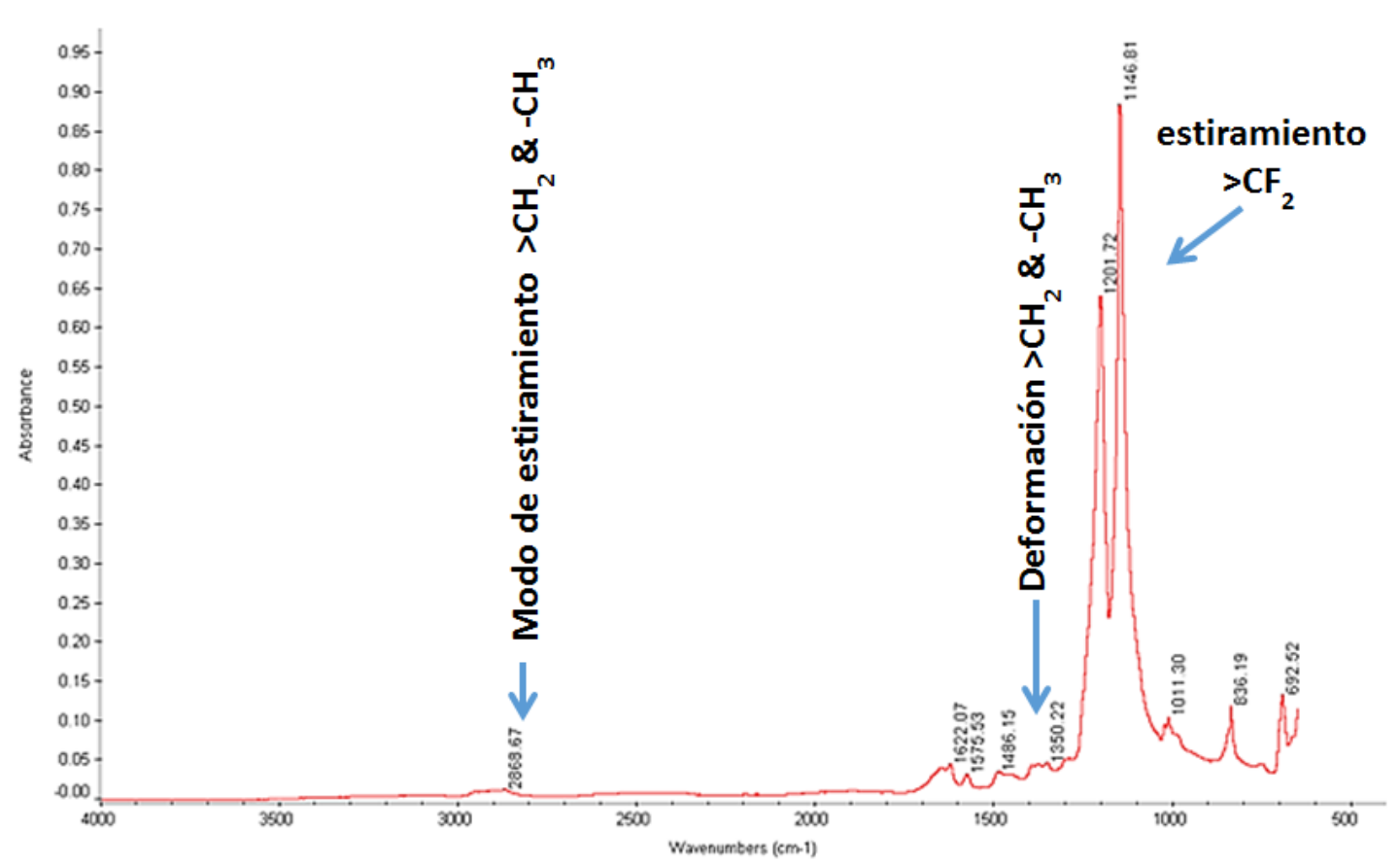

Figura 4. A. La figura indica en rojo el espectro FT-IR para PTFE después de 96 h de exposición a la solución KOH a $32 w t \%$.

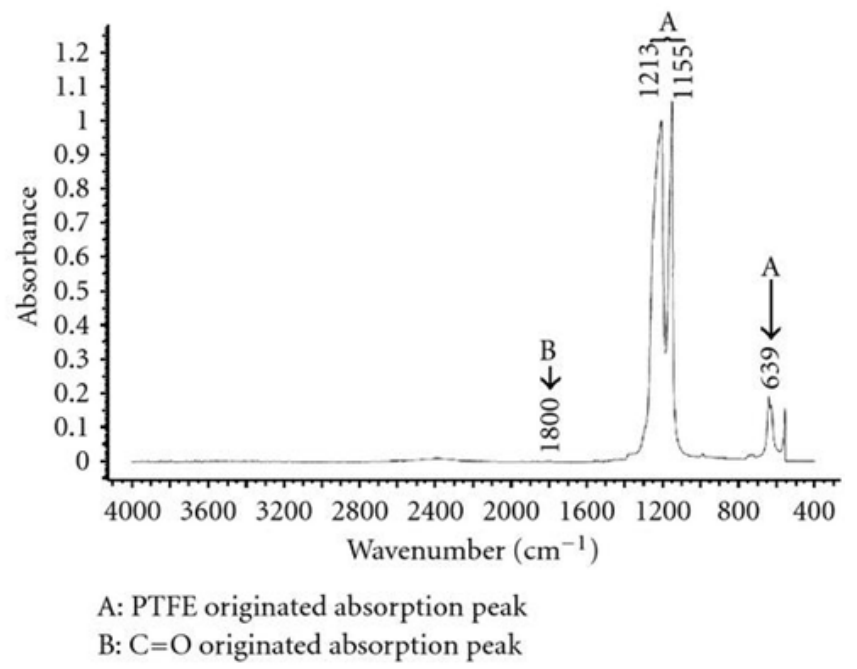

Figura 5. A. La figura indica en negro el espectro de PTFE base (Perkin-Elmer, 2018). Existen diferencias insignificantes entre los dos espectros, demostrando que el PTFE no sufre degradación química.

LA GRANJa: Revista de Ciencias de la Vida 29(1) 2019:17-31.

(C)2019, Universidad Politécnica Salesiana, Ecuador. 